\title{
K-Sol corneal preservation at room temperature
}

\author{
KOICHI TAMAKI, EMILY D VARNELL, AND HERBERT E KAUFMAN \\ From the Lions Eye Research Laboratories, LSU Eye Center, Louisiana State University Medical Center \\ School of Medicine, New Orleans, Louisiana, USA
}

SUMMARY K-Sol, a recently developed corneal storage medium that contains purified chondroitin sulphate in tissue culture medium (TC 199), is capable of preserving corneal tissue for 14 days at $4^{\circ} \mathrm{C}$. To study the effect of tissue storage in K-Sol at room temperature we preserved rabbit corneas in $\mathrm{K}-\mathrm{Sol}$ and $\mathrm{M}-\mathrm{K}$ medium for three or seven days at $25^{\circ} \mathrm{C}$. All of the corneal endothelial sheets were intact after three days. At seven days the change in $\mathrm{pH}$ of the K-Sol medium was less than that of M-K medium. Corneas preserved in M-K medium showed swelling of mitochondria and a decrease in the number of cytoplasmic organelles. Corneas preserved in K-Sol had organised cytoplasmic organelles and nuclei. Scanning electron micrographs revealed well preserved endothelial sheets. Corneas stored in the two media showed no significant difference in thickness. A pair of human corneas preserved in K-Sol at room temperature for six days maintained about $95 \%$ of the endothelial sheet in good condition. Small separations were observed between some of the endothelial cells. However, even in these areas, the cytoplasmic organelles were well preserved. It appeared that $\mathrm{K}-\mathrm{Sol}$ is more stable than $\mathrm{M}-\mathrm{K}$ medium at room temperature, and that both rabbit and human corneas can be preserved in good condition in K-Sol for at least six days at $25^{\circ} \mathrm{C}$.

Kaufman and others developed a new corneal preservation medium (K-Sol) for storing corneal tissue for at least two weeks at refrigerator temperature. ${ }^{1-3} \mathrm{~K}$-Sol contains $2.5 \%$ purified chondroitin sulphate in tissue culture medium (TC-199), 0.025 M HEPES buffer, and gentamicin sulphate. The $\mathrm{pH}$ is 7.4 and the osmolality is 310 mosm. Refrigerator preservation is simple and inexpensive; however, temperature control is uncertain during shipping. At temperatures higher than $4^{\circ} \mathrm{C}$ the metabolic activity of the cornea increases, and the survival of the endothelial cells is threatened by the release of metabolic wastes into the medium. ${ }^{4}$ We examined the stability of K-Sol, as well as the ability of this medium to preserve rabbit and human corneas, at room temperature.

\section{Material and methods}

Animal care and treatment in this investigation were in compliance with the ARVO resolution on the use of animals in research. Twelve New Zealand albino rabbits were killed with an overdose of sodium Correspondence to Herbert E Kaufman, MD, LSU Eye Center, 2020 Gravier Street, Suite B, New Orleans, LA 70112, USA. pentobarbital. The eyes were immediately enucleated and the globes flooded with gentamicin sulphate.

The corneas were sectioned from the eyeball with a 2 to $3 \mathrm{~mm}$ scleral rim and placed epithelial side down. All procedures were carried out by sterile technique in a laminar flow hood. The right corneas were preserved in $\mathrm{K}$-Sol and the mate corneas were preserved in $\mathrm{M}-\mathrm{K}$ medium, both at room temperature $\left(25^{\circ} \mathrm{C}\right)$ for three or seven days (six pairs each). The media were not changed during this study. When the corneas were removed from the medium, the $\mathrm{pH}$ of the medium was measured. The corneas were fixed in $2.5 \%$ glutaraldehyde and $3 \%$ formalin with $0.1 \mathrm{~N}$ sodium cacodylate trihydrate buffer $(\mathrm{pH} 7 \cdot 35)$ for 12

Table 1 Rabbit corneal thickness after preservation at $25^{\circ} \mathrm{C}$

\begin{tabular}{llll}
\hline $\begin{array}{l}\text { Preservation } \\
\text { time }\end{array}$ & \begin{tabular}{l} 
Corneal thickness* $(\mathrm{mm})($ mean $\pm S D)$ \\
\cline { 2 - 3 }
\end{tabular} & $\begin{array}{l}\text { Statistically } \\
\text { significant } \\
\text { difference }\end{array}$ \\
\hline 3 days & $0 \cdot 33 \pm 0.03(\mathrm{n}=8)$ & $0 \cdot 34 \pm 0 \cdot 02(\mathrm{n}=4)$ & No \\
7 days & $0 \cdot 41 \pm 0.02(\mathrm{n}=4)$ & $0 \cdot 42 \pm 0.06(\mathrm{n}=4)$ & No \\
\hline
\end{tabular}

${ }^{*}$ Control thickness $=0.34 \mathrm{~mm}$. 


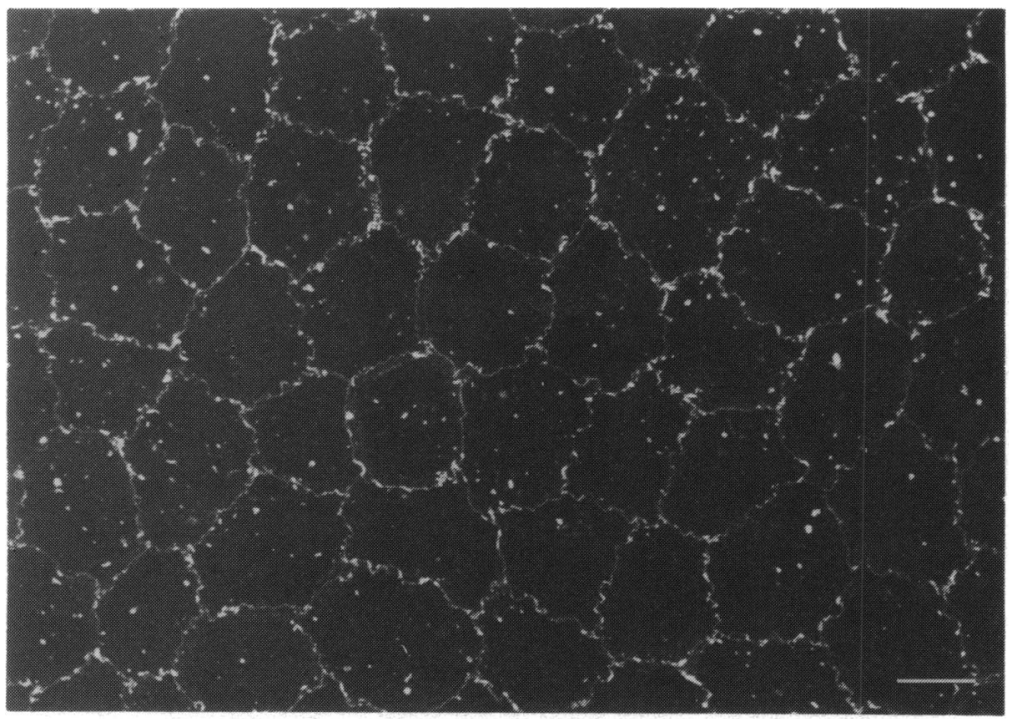

Fig. 1A Scanning electron micrograph of rabbit corneal endothelial cells preserved in $\mathrm{K}$-Sol for three days at room temperature. Normal mosaic-like endothelial cells are seen. The endothelial surface is smooth and the overlapping interdigitations can be seen clearly. Bar $=10 \mu \mathrm{m}$.

hours, washed in the same buffer, postfixed in buffered $1 \%$ osmium tetroxide for 90 minutes, washed again in buffer, and dehydrated in graded alcohols from $50 \%$ to $100 \%$ and propylene oxide.

Half of each cornea was embedded in Epon. Embedded specimens were sectioned with a microtome and stained with toluidine blue for light microscopy or uranyl acetate and lead citrate for transmission electron microscopy. Corneal thickness was measured on light micrographs which were enlarged 100 times.

For scanning electron microscopy the other half of each cornea was fixed in $2.5 \%$ glutaraldehyde and $3 \%$ formalin with $0 \cdot 1 \mathrm{~N}$ sodium cacodylate trihydrate buffer ( $\mathrm{pH} 7.35)$ for 12 hours, dehydrated in alcohol, dried in a critical point dryer, placed on aluminium stubs, coated with gold, and photographed.

A pair of corneas from a 33-year-old human donor was obtained 2 hours and 25 minutes post mortem, placed in K-Sol by the eye bank, and stored at room temperature for six days. The corneas were prepared for scanning electron microscopy and transmission electron microscopy, and corneal thickness was measured as described above.

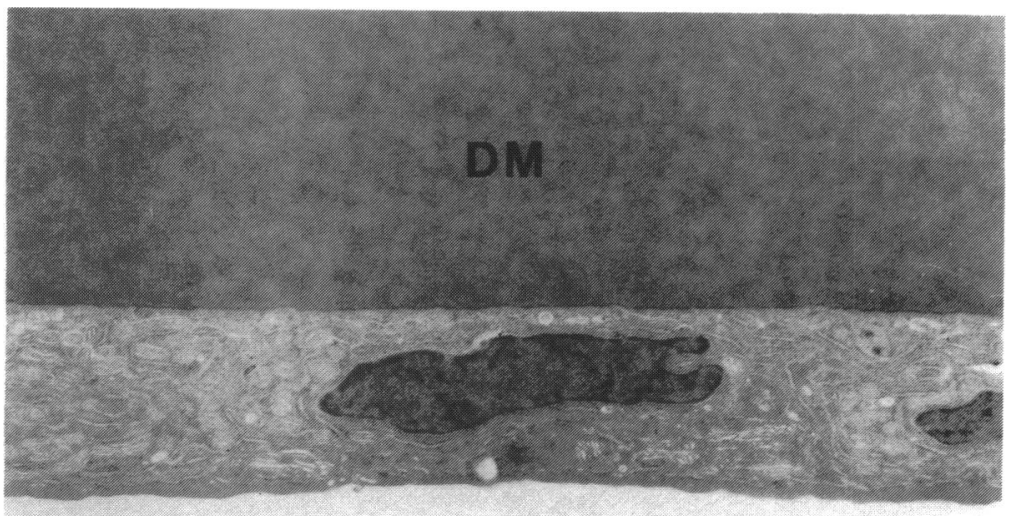

Fig. 1B Transmission electron micrograph of the same cornea. The nucleus and cytoplasmic organelles are preserved intact. Bar $=1 \mu \mathrm{m}$. 
Fig. 2A Scanning electron micrograph of rabbit corneal endothelial cells preserved in $\mathrm{K}$-Sol for seven days. The mosaic-like pattern of the endothelial sheets can be seen. The lateral cytoplasmic borders are comparatively distinct. Cellular pleomorphism is visible and the endothelial surface is ruffled. Bar $=10 \mu \mathrm{m}$.

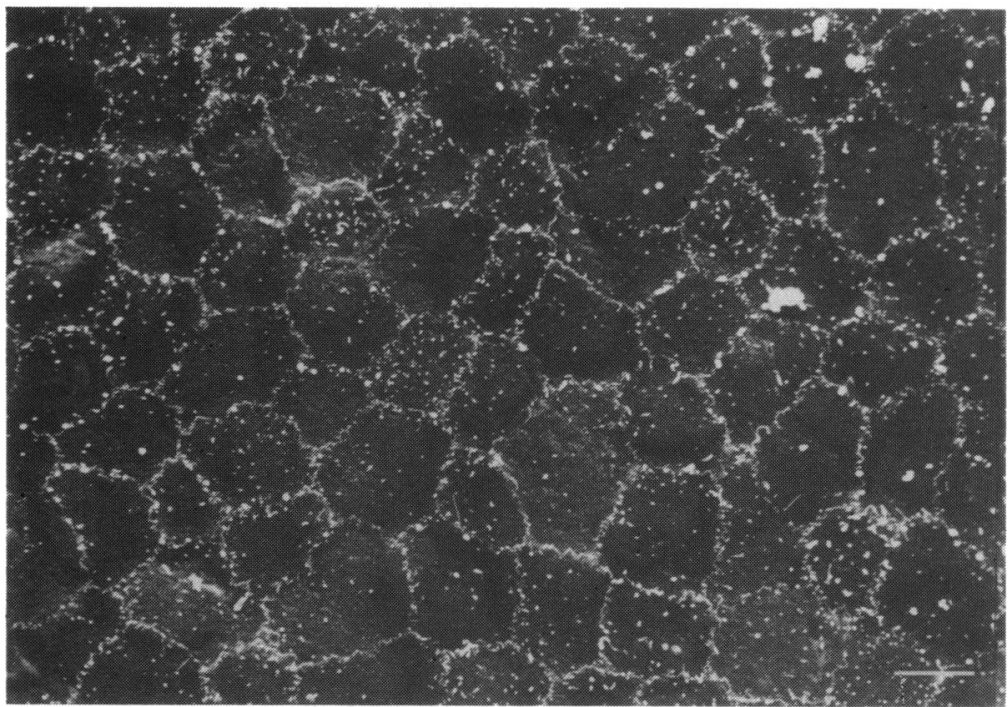

\section{Results}

\section{PH AND CORNEAL THICKNESS}

The $\mathrm{pH}$ was measured in two samples of each medium at three and seven days. Both $\mathrm{K}-\mathrm{Sol}$ and M-K medium had a $\mathrm{pH}$ of 7.40 before use. The average $\mathrm{pH}$ was $7 \cdot 28$ for the $\mathrm{K}$-Sol and $7 \cdot 15$ for $\mathrm{M}-\mathrm{K}$ medium at three days. After seven days the average $\mathrm{pH}$ was 7.17 for K-Sol and 7.04 for M-K medium. The average $\mathrm{pH}$ of the $\mathrm{K}$-Sol in which the human corneas were stored was $\mathbf{7 \cdot 2 7}$ after six days.

At three days the average thickness of the corneas stored in K-Sol was $0.33 \mathrm{~mm}$ (SD 0.03) and of the corneas stored in M-K medium $0.34 \mathrm{~mm}$ (SD 0.02).
After seven days, corneal thickness was $0.41 \mathrm{~mm}$ (SD $0.04)$ in the K-Sol preserved corneas and $0.42 \mathrm{~mm}$ (SD 0.06) in the M-K medium preserved corneas. The difference was not statistically significant (Table 1).

\section{HISTOLOGY}

The appearance of the endothelial cells was similar in all specimens. The rabbit corneas preserved in K-Sol for three days at room temperature showed normal mosaic-like endothelial sheets with normal overlapping interdigitations of the cells (Fig. 1A). The cytoplasmic organelles (mitochondria, Golgi apparatus, and rough endoplasmic reticulum) were

Fig. 2B Transmission electron micrograph of the same cornea. The thickness of the endothelial cells is increased $(4 \cdot 7 \mu \mathrm{m})$. The cytoplasm is less dense anterior to the nucleus. The cytoplasmic organelles and nucleus are preserved in good condition. Bar $=1$ um.

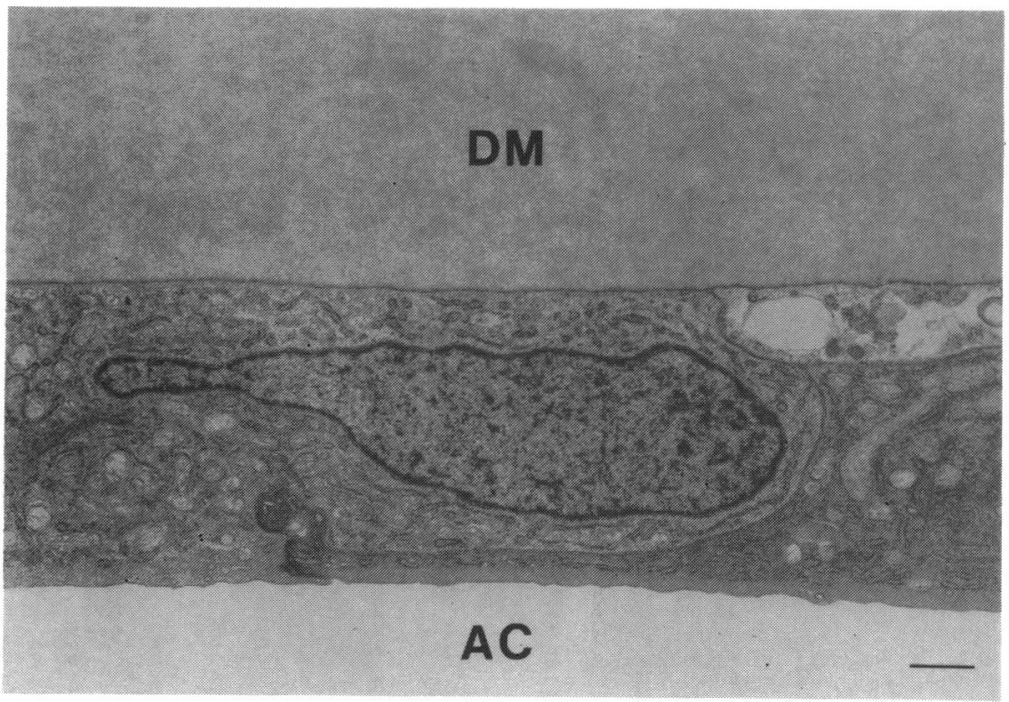




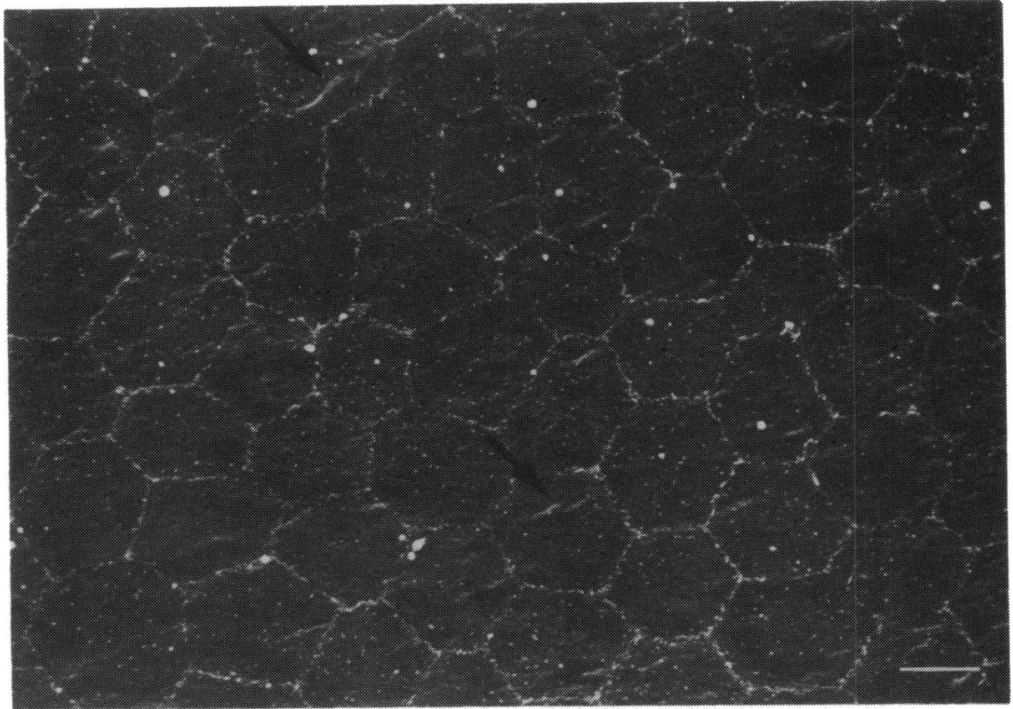

Fig. 3A Scanning electron micrograph of rabbit corneal endothelial cells preserved in $M-K$ medium for three days at room temperature. Normal mosaic-like endothelial sheets are seen. Some of the endothelial surface is ruffled (arrows). Bar $=10 \mu \mathrm{m}$.

intact, and the nucleus was dense (Fig. 1B). With seven-day preservation the mosaic-like pattern of the endothelial sheets could be seen, and the lateral cell borders were relatively distinct. Cellular pleomorphism and ruffling of the endothelial surface were visible (Fig. 2A). Compared with the corneas stored for three days, which were $3.2 \mu \mathrm{m}$ thick, the corneas stored for seven days showed an increase in corneal thickness to $4.7 \mu \mathrm{m}$, and the cytoplasmic density was somewhat decreased anterior to the nucleus. However, the endothelial cells were organised and appeared to be in good condition (Fig. 2B).

The rabbit corneas preserved in $\mathrm{M}-\mathrm{K}$ medium for three days at room temperature showed normal mosaic-like endothelial sheets. Some of the endothelial surfaces were slightly ruffled (Fig. 3A). Transmission electron microscopy revealed intact endothelial cells. The density of the cytoplasmic organelles was slightly decreased anterior to the nucleus (Fig. 3B). After seven days of preservation, mosaic-like hexagonal endothelial sheets, as well as cellular pleomorphism and ruffling of the surface membrane, were seen (Fig. 4A). Transmission electron microscopy revealed a decrease in the numbers of cytoplasmic organelles, and the mitochondria were swollen. These changes were more prominent anterior to the nucleus (Fig. 4B).

The human corneas preserved in K-Sol for six days

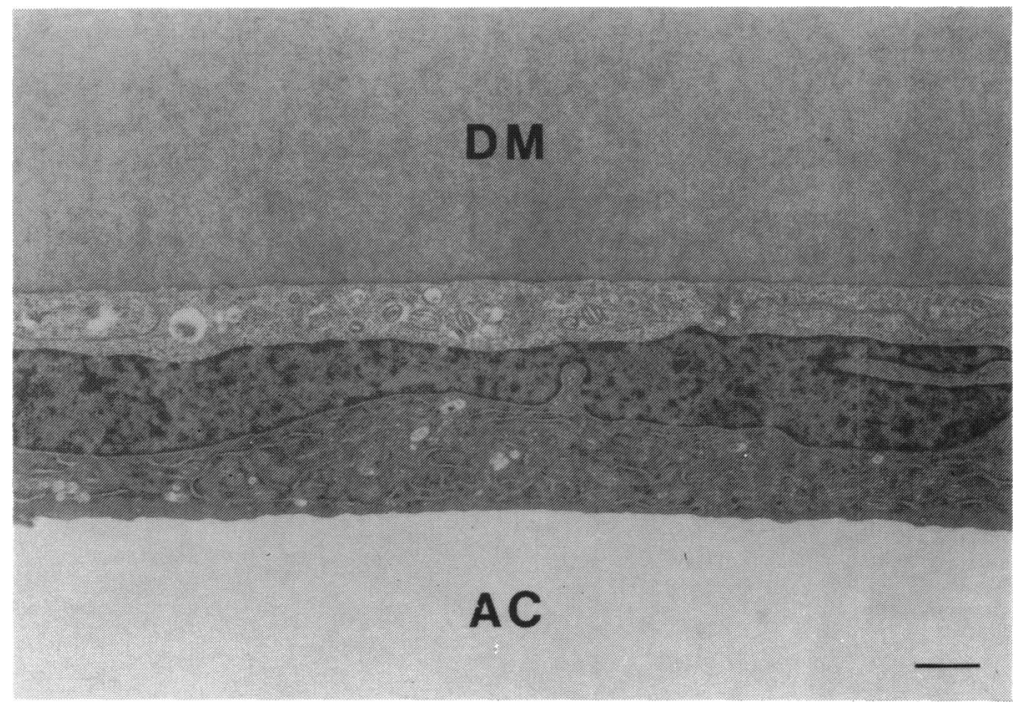

Fig. 3B Transmission electron micrograph of the same cornea. Cytoplasmic organelles anterior to the nucleus are fewer in number and small vacuolations can be seen. The endothelial cells are almost intact. Bar $=1$ um. 
Fig. 4A Scanning electron micrograph of rabbit corneal endothelial cells preserved in $M-K$ medium for seven days at room temperature. Mosaic-like hexagonal endothelial sheets, cellular pleomorphism, and ruffling of the endothelial surface can be seen. Bar $=10 \mu \mathrm{m}$.

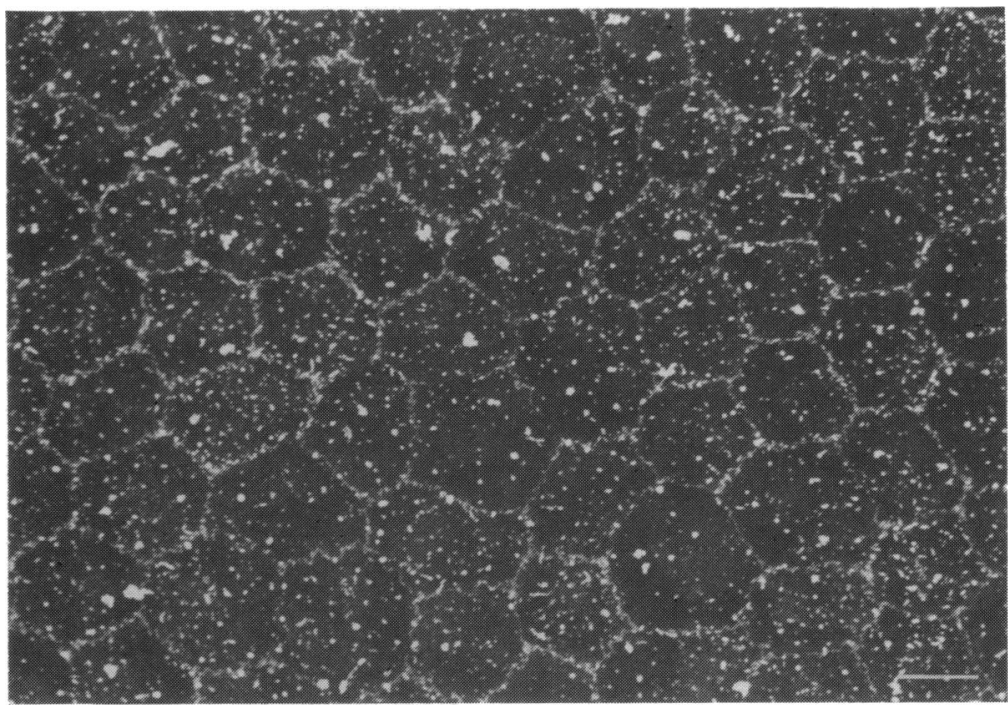

\section{Discussion}

Rabbit corneal endothelial cells were ultrastructurally intact after storage for three days at room temperature in both $\mathrm{K}$-Sol and $\mathrm{M}-\mathrm{K}$ medium. At seven days corneas preserved in K-Sol were still in good condition. The cytoplasmic organelles and nuclei were well preserved. However, corneas preserved in $\mathrm{M}-\mathrm{K}$ medium showed mitochondrial swelling and fewer cytoplasmic organelles. These histological observations suggest that K-Sol preserves rabbit corneas better than $\mathrm{M}-\mathrm{K}$ medium at room temperature. In addition the $\mathrm{pH}$ of $\mathrm{K}$-Sol was more stable than that of M-K medium, although both
Fig. 4B Transmission electron micrograph of the same cornea. The endothelial cells are thicker $(5 \cdot 1 \mu \mathrm{m})$ than the cells on corneas stored in $M-K$ medium for three days $(3.5 \mu \mathrm{m})$. Fewer cytoplasmic organelles are seen, and mitochondrial swelling is prominent anterior to the nucleus. $B a r=1 \mu \mathrm{m}$.

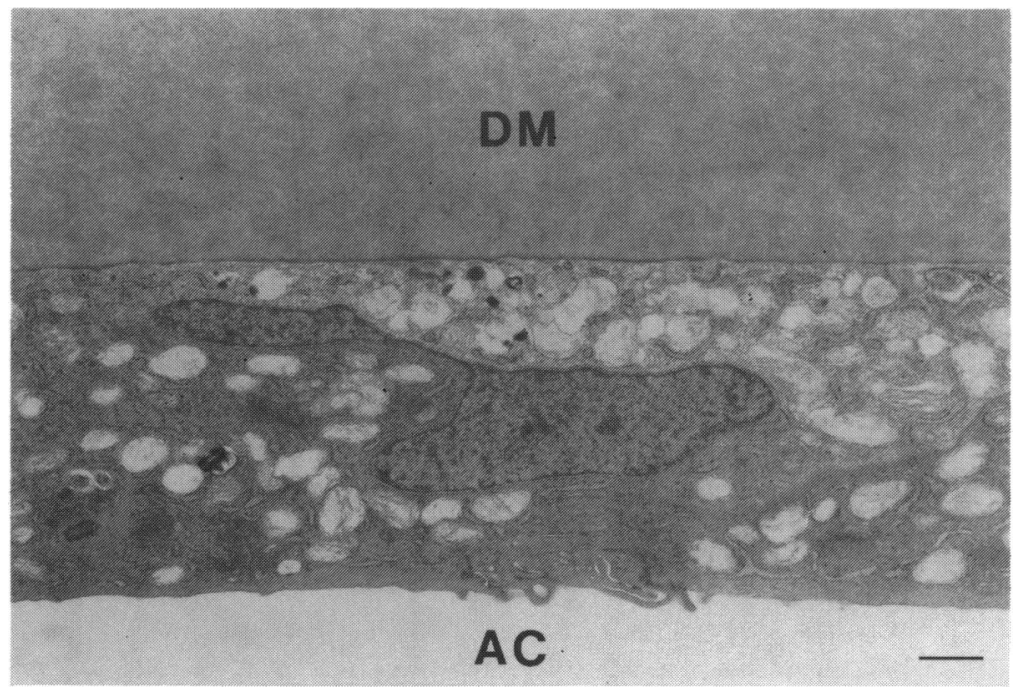






Fig. 5A Scanning electron micrograph of the human corneal endothelial cells preserved in $\mathrm{K}$-Sol for six days at room temperature. The mosaic-like pattern of the endothelial sheets can be seen. The lateral cytoplasmic membranes are indistinct. Some of the cytoplasmic membranes protrude along the lateral cytoplasmic membranes. Bar $=10 \mu \mathrm{m}$.

media maintained $\mathrm{pH}$ in the optimum range $(6 \cdot 8$ to $8 \cdot 2)^{5}$ for maintenance of corneal endothelial integrity and function.

Sachs and his colleagues ${ }^{4}$ reported disorganised cytoplasmic organelles in human corneas preserved for four days in M-K medium at room temperature. In our study human corneal endothelial cells preserved in K-Sol medium at room temperature were still in good condition after six days. The cytoplasmic organelles and nuclei were intact. Scanning electron microscopy revealed a few protrusions on the surface of about $5 \%$ of the endothelial cells (Fig. 5A), which corresponded to small intercellular separations seen by transmission electron microscopy (Fig. 5C). How- ever, the cytoplasmic organelles were still well preserved even in the protruding areas. It appears that K-Sol preserves human corneas better than M-K medium at room temperature for six days.

There was no significant difference in corneal thickness between $\mathrm{K}$-Sol and M-K medium preserved rabbit corneas. No stromal swelling was seen in these or in the human corneas preserved in K-Sol. Recently Lindstrom and others ${ }^{6}$ reported that chondroitin sulphate prevents stromal swelling and preserves corneal endothelial cells in organ culture. K-Sol, which contains purified chondroitin sulphate, demonstrates this ability also in room temperature preservation.

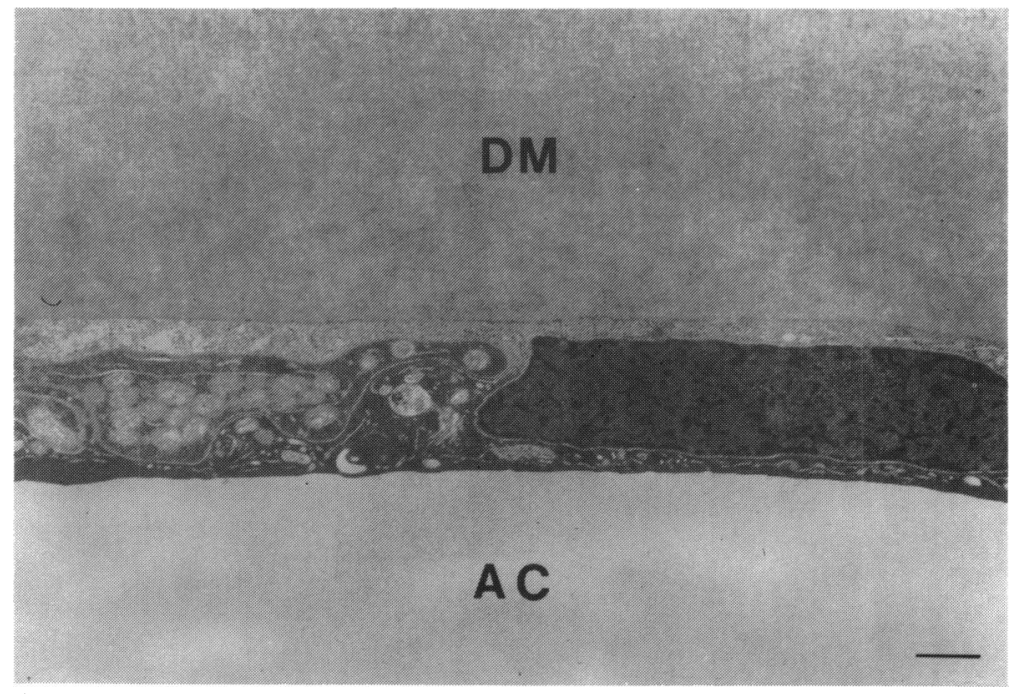

Fig. 5B Transmission electron micrograph of the same cornea. Endothelial cells are attenuated (2-3 $\mu \mathrm{m})$. Almost all endothelial cells show intact cytoplasmic organelles and nuclei. Bar $=1$ um. 
Fig. 5C Transmission electron micrograph of the human corneal endothelial cells. Small intercellular separations $\left(^{*}\right)$ can be seen between some of the endothelial cells, but the cytoplasmic organelles in these cells are still well preserved. Bar $=1 \mu \mathrm{m}$.



Previously Lindstrom and his colleagues? suggested that room temperature preservation eliminates the need for an incubator or refrigerator, reducing the expense and complexity of temperature maintenance. However, room temperature preservation requires a completely sterile technique to prevent infection. For this reason refrigerator preservation seems safer and easier than room temperature preservation.

We are not suggesting that room temperature preservation be considered as an alternative to the widespread practice of refrigerator storage of donor corneal tissue, but we have shown here that tissue stored in K-Sol can successfully withstand some period of time at room temperature and remain intact. The ability to preserve corneas at room temperature would make the shipping of donor tissue for long distances and to areas without reliable refrigeration easier and safer, thereby increasing the utilisation of available tissue and reducing wastage.
This study was supported in part by Public Health Service grants EY02580 and EY02377 from the National Eye Institute, National Institutes of Health, Bethesda, Maryland.

\section{References}

1 Kaufman HE, Varnell ED, Kaufman S. Chondroitin sulfate in a new corneal preservation medium. Am J Ophthalmol 1984; 98: $112-4$.

2 Kaufman HE, Varnell ED, Kaufman S, Beuerman RW, Barron BA. K-Sol corneal preservation. Am J Ophthalmol 1985; 100: 299-304.

3 Yau CW, Kaufman HE. A medium-term corneal preserving medium (K-Sol). Arch Ophthalmol 1986; 104: 598-601.

4 Sachs V, Goldman K, Valenti J, Kaufman HE. Corneal storage at room temperature. Arch Ophthalmol 1978; 96: 1075-7.

5 Edelhauser HF, Gonnering R, Van Horn DL. Intraocular irrigating solutions. Arch Ophthalmol 1978; 96: 516-20.

6 Lindstrom RL, Skelnik DL, Mindrup EA, Doughman DJ. Organ culture corneal preservation with chondroitin sulfate. ARVO abstract. Invest Ophthalmol Vis Sci 1984; 25 (suppl): 266.

7 Lindstrom RL, Doughman DJ, Van Horn DL, Schmitt MK, Byrnes PJ. Organ culture corneal storage at ambient room temperature. Arch Ophthalmol 1977; 95: 869-78.

Accepted for publication 12 March 1987. 